\title{
The Effect of Androgen on Fine Structure of the Prostate of Castrated Rats
}

\section{Report II. The Effect by Administration of Small Dosis of Testosterone in 3 Days Soon after Castration}

\author{
By \\ Tadao Kanai \\ From the Department of Obstetrics and Gynecology, Tohoku \\ University School of Medicine, Sendai; \\ Director: Prof. K. Kushima"
}

(Received for publication, August 15, 1961)

\section{INTRODUCTION}

In the previous study, ${ }^{1}$ the present author has reported on his findings of the prostatic epithelial cells of rats after 7 days' administration of testosterone one month after castration, when the effect of castration had already become evident. The results showed that the cells could not be perceptibly recovered from the atrophy caused by being left untreated for a month by administration of small doses of $1-10 \mu \mathrm{g}$., but the picture of atrophy was considerably effaced with $100 \mu \mathrm{g}$. and above of testosterone. So, this time, the cells were subjected to electron microscopic examination after giving testosterone in the early stage of change due to castration. The following results were obtained.

\section{MATERIALS AND METHODS}

Eight pure Wistar-breed male castrated rats, weighing about $140 \mathrm{~g}$., were divided into the following 4 groups: Group I of controls, left untreated for 4 days after castration; groups II, III and IV received 1, 10 and $100 \mu \mathrm{g}$. of testosterone, respectively, in 3 days.

Testosterone was administered in the form of solution in olive oil, containing 1,10 and $100 \mu \mathrm{g}$. each in $0.3 \mathrm{ml}$, of the oil, by injection of $0.1 \mathrm{ml}$. daily beneath the dorsal skin. The injection started on the day of castration, and these animals were examined 24 hours after the last injections. The methods of excision, fixation and microscopy were the same as in report I.

\section{RESULTS}

1. Findings in group I (Figs. 1, 2 and 3)

The prostatic epithelial cells (PEC hereunder) were ranged in one to several

金井忠男 
layers lining the lumen, and the height of cells in this group was lower than in the normal PEC. The microvilli were ranged somewhat irregularly and decreased in number in some cells. In the supranuclear portion, the rough-surfaced endoplasmic reticula looked lamellar or vortical in appearance and were supernormally dilated, in some places in lake-like expanse, but atrophied endoplasmic reticula with reduced sacs were also observable. The mitochondria were somewhat subnormal in number, but their inner structure was mostly clearcut. The Golgi's complexes were dilated and round vesicles were conspicuous. The number of round granules with high electron density, presumably lipoid granules, were somewhat supernormal. The nucleus was somewhat irregular and the nucleoplasm showed slightly the appearance of granules coagulated at its margin. Near the basement membrane, light interstices with fine granules were seen between the cells.

2. Findings in group II (Figs. 4, 5 and 6)

The PEC were taller than in group I. The microvilli were also relatively larger and were arranged regularly, approaching the normal picture. In the supranuclear portion the endoplasmic reticula were ranged in lamellae and dilated in some places, especially, into spheres near the luminar border. The mitochondria were somewhat small-sized and the cristae often lacked in definition. The Golgi's complexes were well developed, vesicles and granules being in evidence. Dense round granules of somewhat subnormal size were found near the luminar border.

3. Findings in group III (Figs. 7 and 8)

The PEC were still taller than in the preceding. The endoplasmic reticula, though dilated in some places, were nearly normal in general appearance, and the Palade's particles were also well defined. The mitochondria were mostly rodlike and contained ill-defined cristae. Dense round granules, some of high and some of low electron density, were ranged near the luminar border.

4. Findings in group IV (Figs. 9 and 10)

The PEC were of remarkable tallness. The nucleus standing near the base and its size being nearly normal, the cytoplasm looked as if greatly increased. The microvilli were well-developed. The well-developed and neatly arranged endoplasmic reticula were found in the supranuclear portion. The sacs of these endoplasmic reticula being dilated in most part, the proper cytoplasm seemed diminished. A part of the sacs of endoplasmic reticula was dilated lakewise. In the interspace between the luminar border and the nucleus of the PEC was found a very well-developed Golgi's complex, containing many enlarged vesicles and granules. Near the luminar border, many granules of more or less high electron density were congregated. The mitochondria were abundant in the intermediate portion, being more frequently rod-like. The nuclei were generally spherical and the nucleoplasmic granules were ranged diffusely. 


\section{DISCUSSION}

In the previous study, by the administration of very small doses of testosterone, no perceptible change detectable under an electron microscope could be evoked in the atrophied prostatic epithelial cells of castrated rats, seemingly because the animals were left untreated too long after castration. Therefore, another study was conducted to examine the effect of administration of hormones following shortly after castration.

As stated above, in the animals left untreated 4 days after castration, dilated and atrophied endoplasmic reticula were found in promiscuous coexistence, the mitochondria were somewhat reduced in size and the dense granules increased in number. When, however, administration of testosterone was begun on the very day of castration, to forestall, if possible, the changes caused by castration, the microvilli came to nearly normal development, the endoplasmic reticula though partly dilated, were also nearly normal, the mitochondria had their cristae less well defined and the round granules increased after only $1 \mu \mathrm{g}$. of testosterone. After $10 \mu \mathrm{g}$. of it, the PEC were still taller, and besides the normalization of the sacs of endoplasmic reticula and enlargement of Golgi's complex, round granules increased especially in the vicinity of the luminar border. After $100 \mu \mathrm{g}$., such changes to the better were further fortified. Harkin ${ }^{2)}$ says that the degree of dilatation of the sacs of the endoplasmic reticula seems to be related to the degree of secretory activity of the cells, and that the dilatation of sacs observable soon after castration is due to the decrease of function of liberation preceding the decrease in production of the secretion. The dilatation of a part of endoplasmic reticulum 4 days after castration in coexistence with atrophy of some of the cells, observed in author's study, may present some difficulty in interpretation, but the tendency to normalization of the sacs after larger doses of hormone seems to support Harkin's opinion.

In proportion with the increase of administered hormone, the dense granules also increased ; this may be also in close relation with the recovery of secretory function. The notable development of Golgi's complex may be also regarded as a natural consequence, seeing that this complex is taken to be an organella partaking in the function of secretion or biosynthesis.

At any rate, the characteristic findings in prostatic epithelial cells by administration of testosterone soon after castration consist in the change in development of endoplasmic reticulum, Golgi's complex and dense granules, and it is probable that these intracellular structures are all closely connected with the secretory function of the cells.

\section{SUMMARY}

The fine structure of the prostatic epithelial cells of mature male rats left 
untreated or treated with testosterone after castration was examined under an electron-microscope on the 4 th day.

1. In the untreated animals, dilated endoplasmic reticula were found in mixture with atrophied reticulum, the mitochondria were somewhat subnormal in size and number, and dense granules were somewhat supernormal in number.

2. In animals given testosterone for 3 days soon after castration, the sacs of the endoplasmic reticulum neared the normal state, the Golgi's complex became better developed and the dense granules increased, with the increase of dosis from 1 to 10 to $100 \mu \mathrm{g}$.

\section{References}

1) Kanai, T., Tohoku J. Exp. Med., 1961, 75, 181.

2) Harkin, J. C., Endocrinology, 1957, 60, 185. 


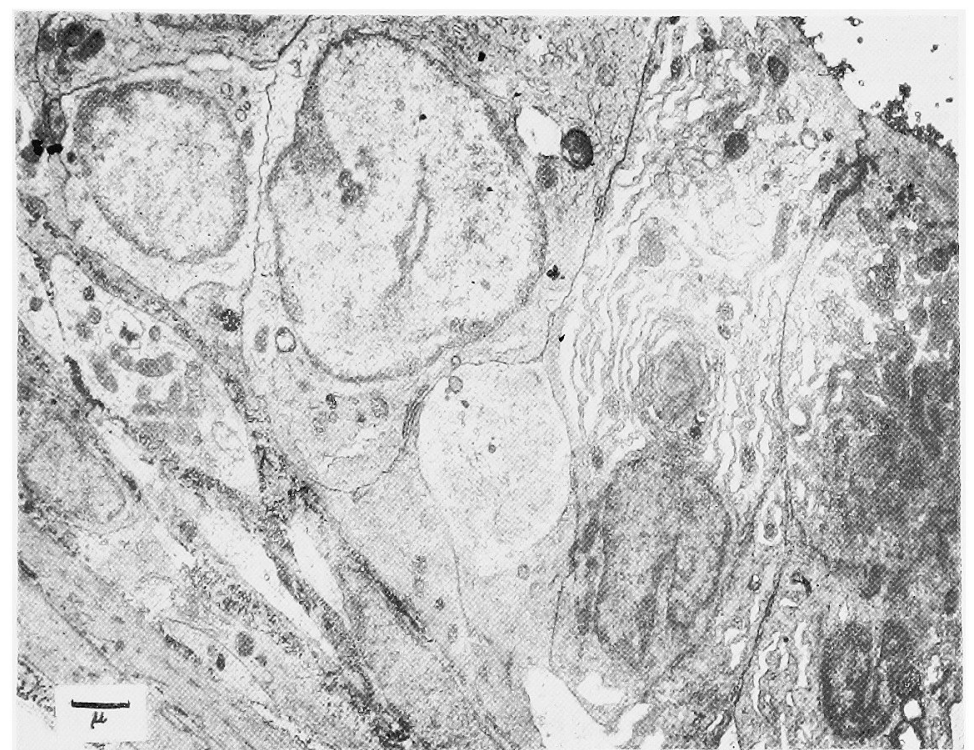

Fig. 1. Prostate left untreated 4 days after castration.

The endoplasmic reticula are slightly enlarged in the right and atrophied in the left cell. Mitochondria are slightly undersized and the cristae are somewhat undefinite (Compare with those in the stromal cells). Light spaces containing fine granules are seen between the cells. Dense granules present.

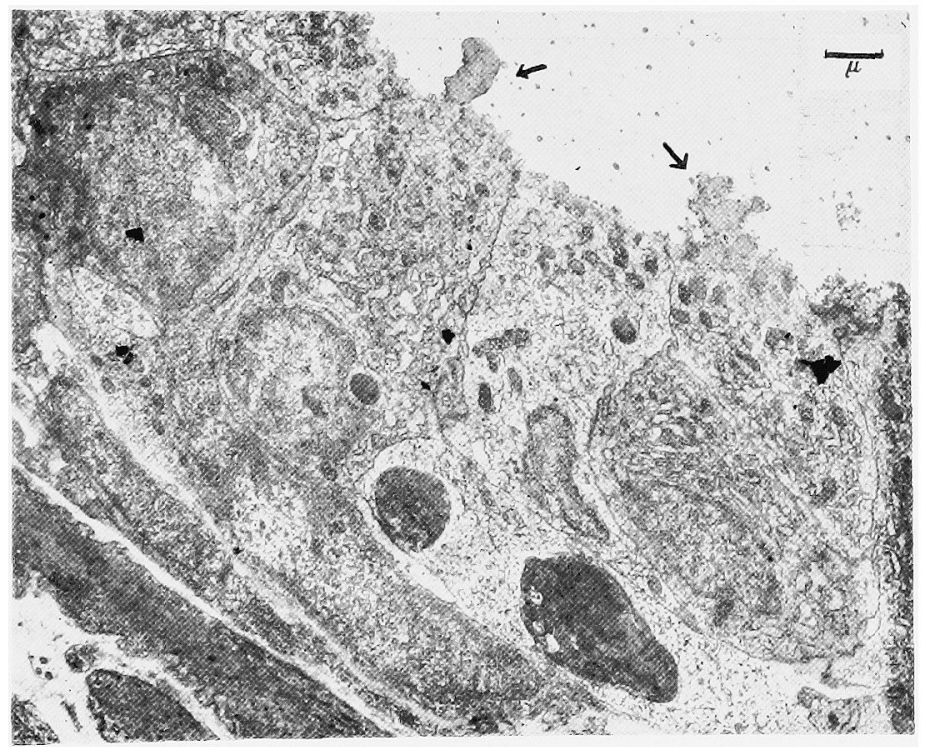

Fig. 2. Ditto.

Findings suggestive of apocrine secretion in 2 places (indicated by arrows). Endoplasmic reticula are somewhat atrophied. Mitochondria are undersized, but cristae are definite. 


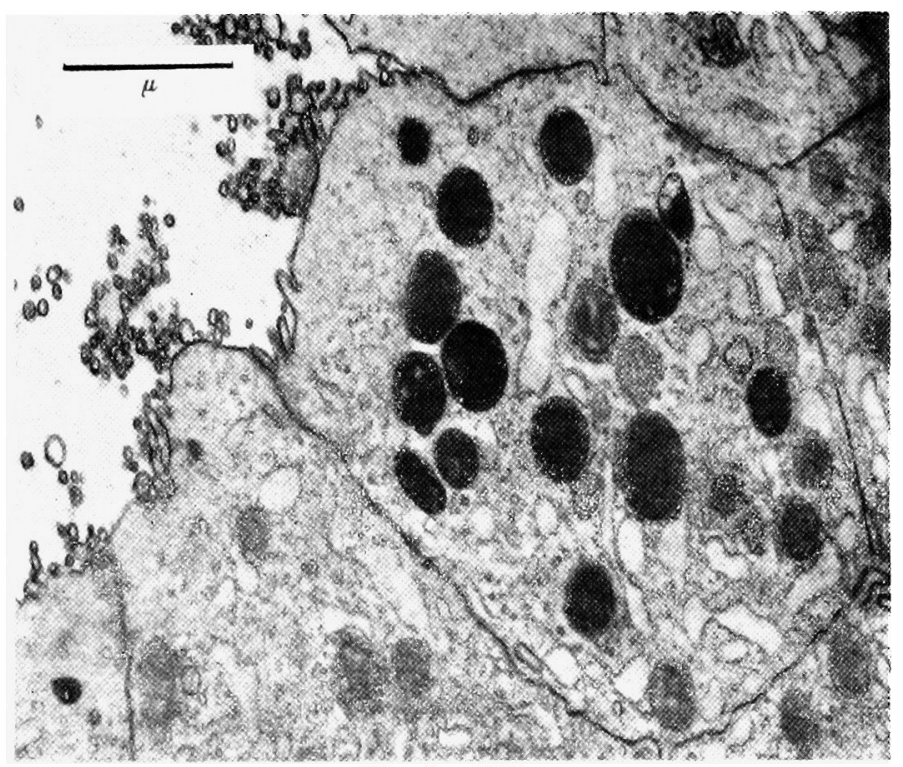

Fig. 3. Ditto.

Dense round gianules, mitochondria and dilated endoplasmic reticulum in mixed coexistence.

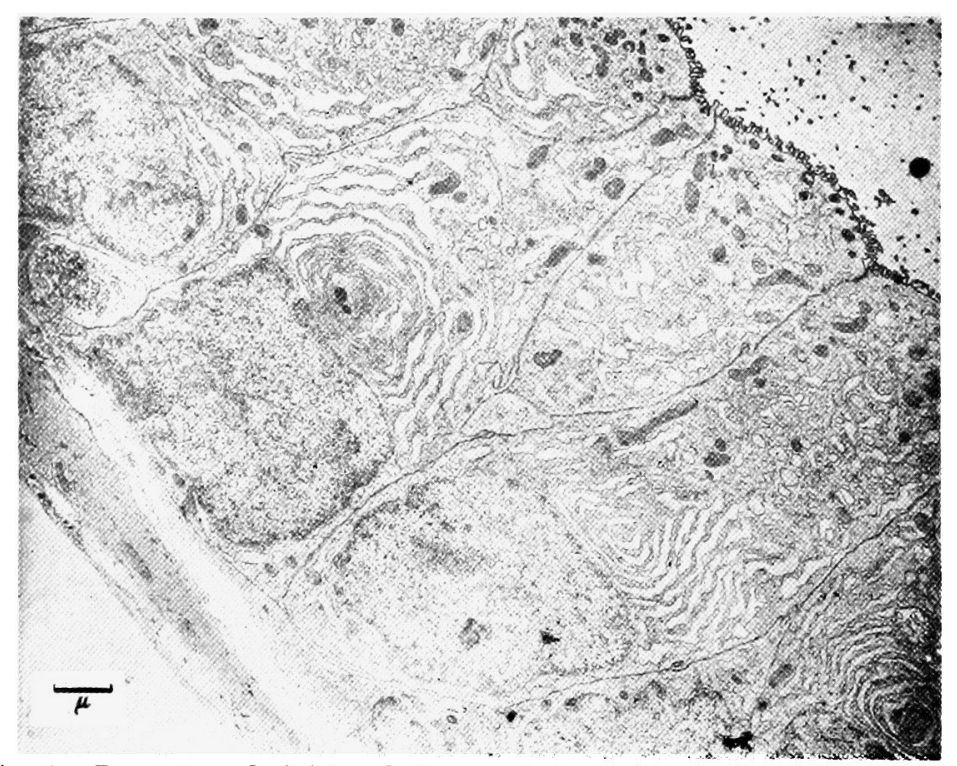

Fig. 4. Prostate administered $1 \mu \mathrm{g}$. of testosterone in 3 days soon after castration.

Endoplasmic reticulum slightly enlarged and Golgi's complex developed. Microvilli nearly normal. 


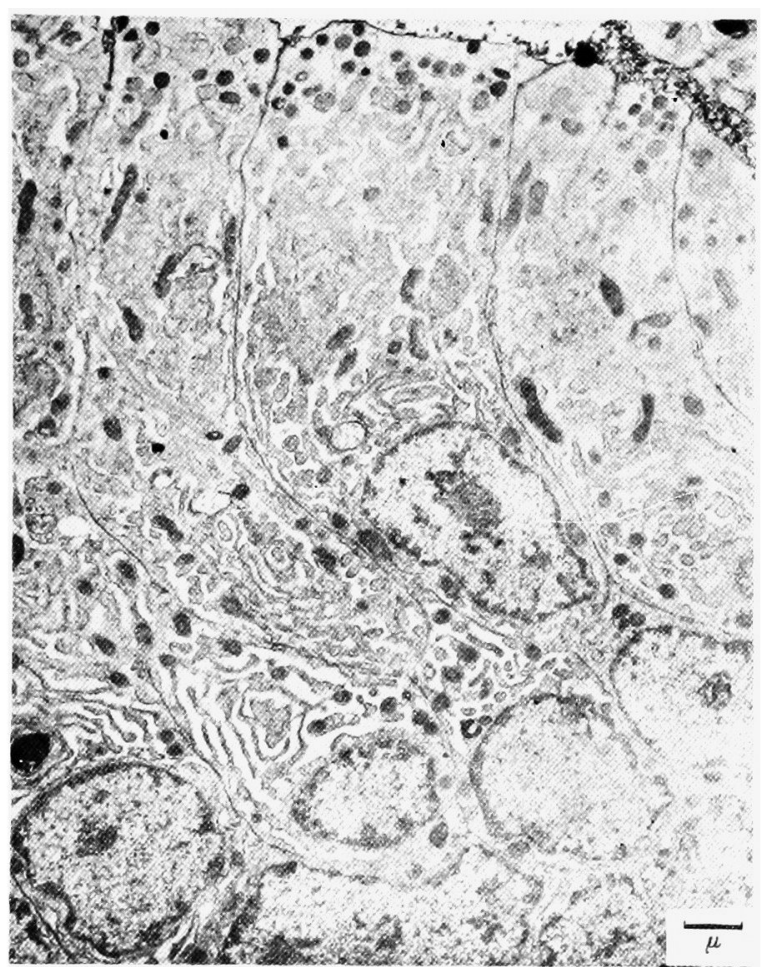

Fig. 5. Ditto. Dense granules near the luminar border.

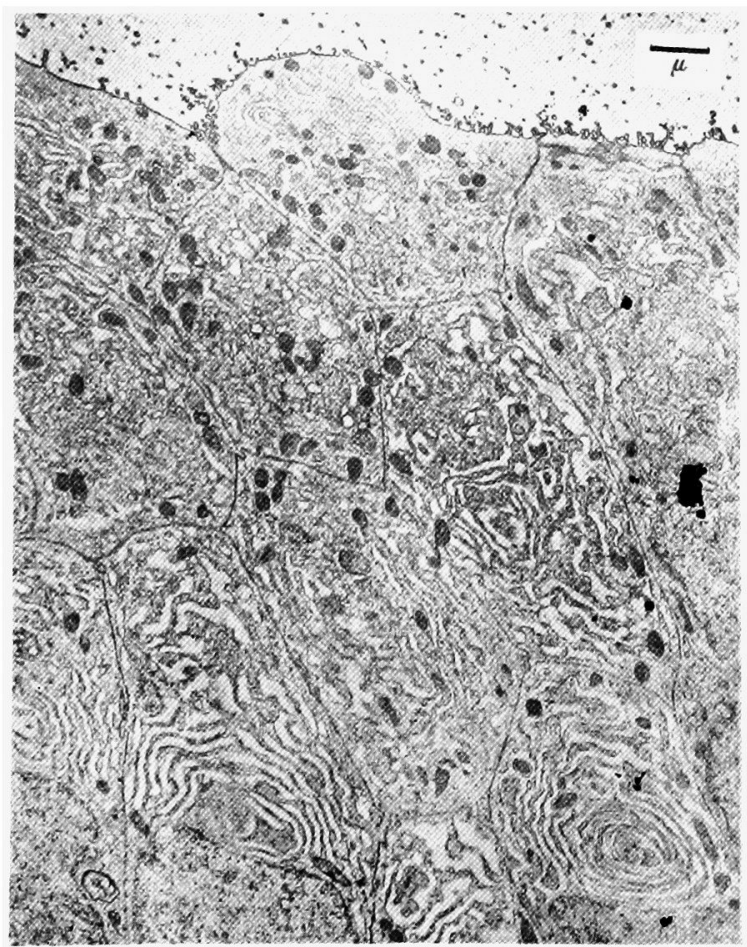

Fig. 6. Ditto. In some places dilated endoplasmic reticulum. 


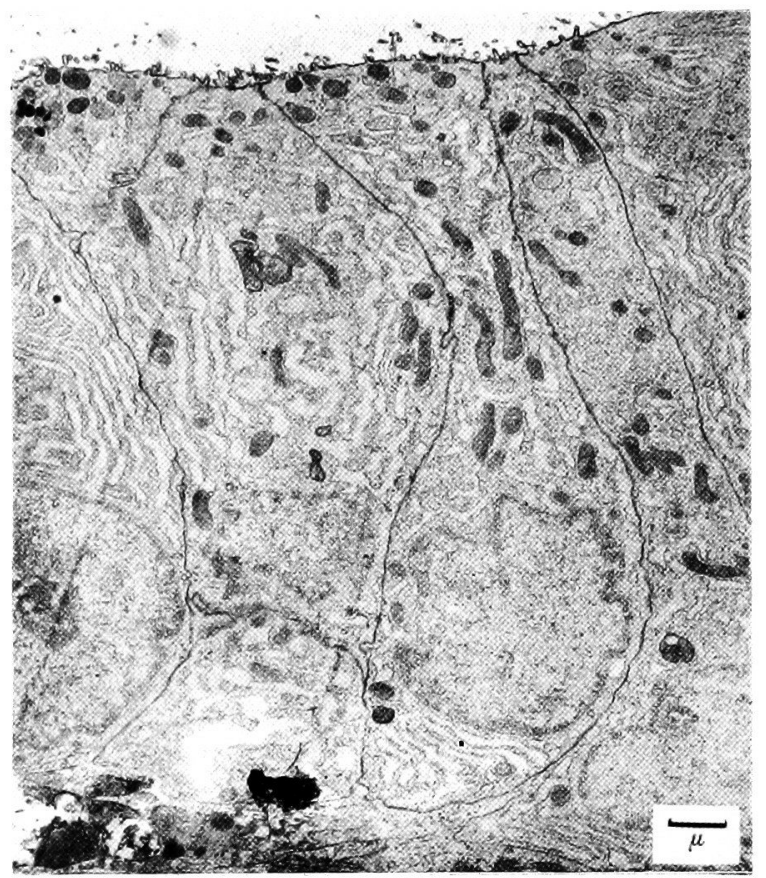

Fig. 7. Prostate administered $10 \mu \mathrm{g}$. of testosterone in 3 days soon after castration.

Round granules of more and less high electron density in mixture. Mitochondria of mostly rod-form and cristae blurred.

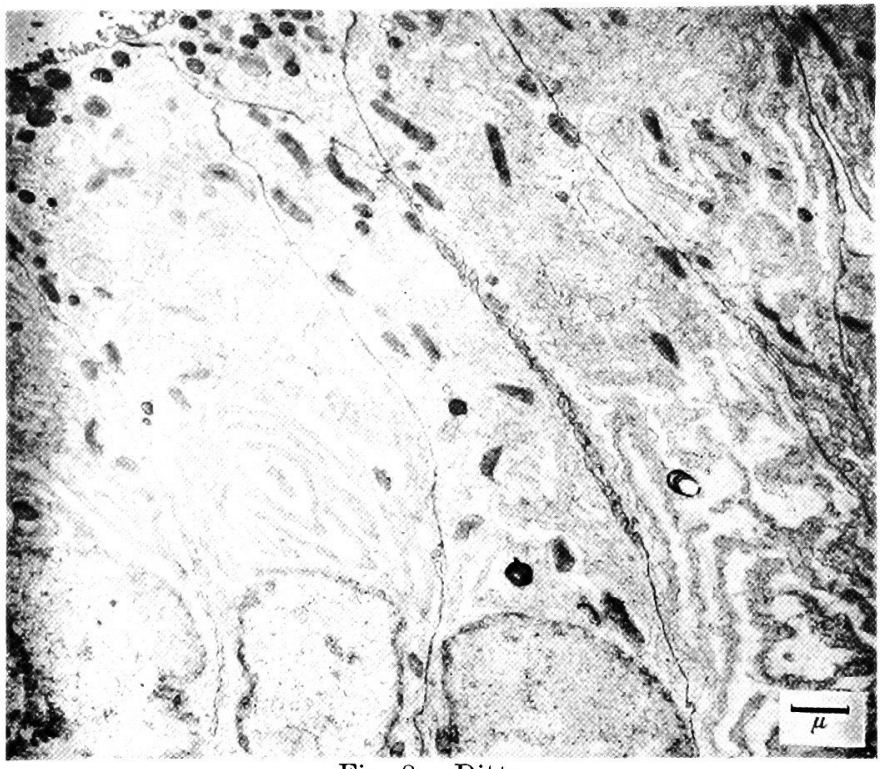

Fig. 8. Ditto.

Golgi's complex enlarged. Endoplasmic reticulum also enlarged in some places. Interdigitation present. 


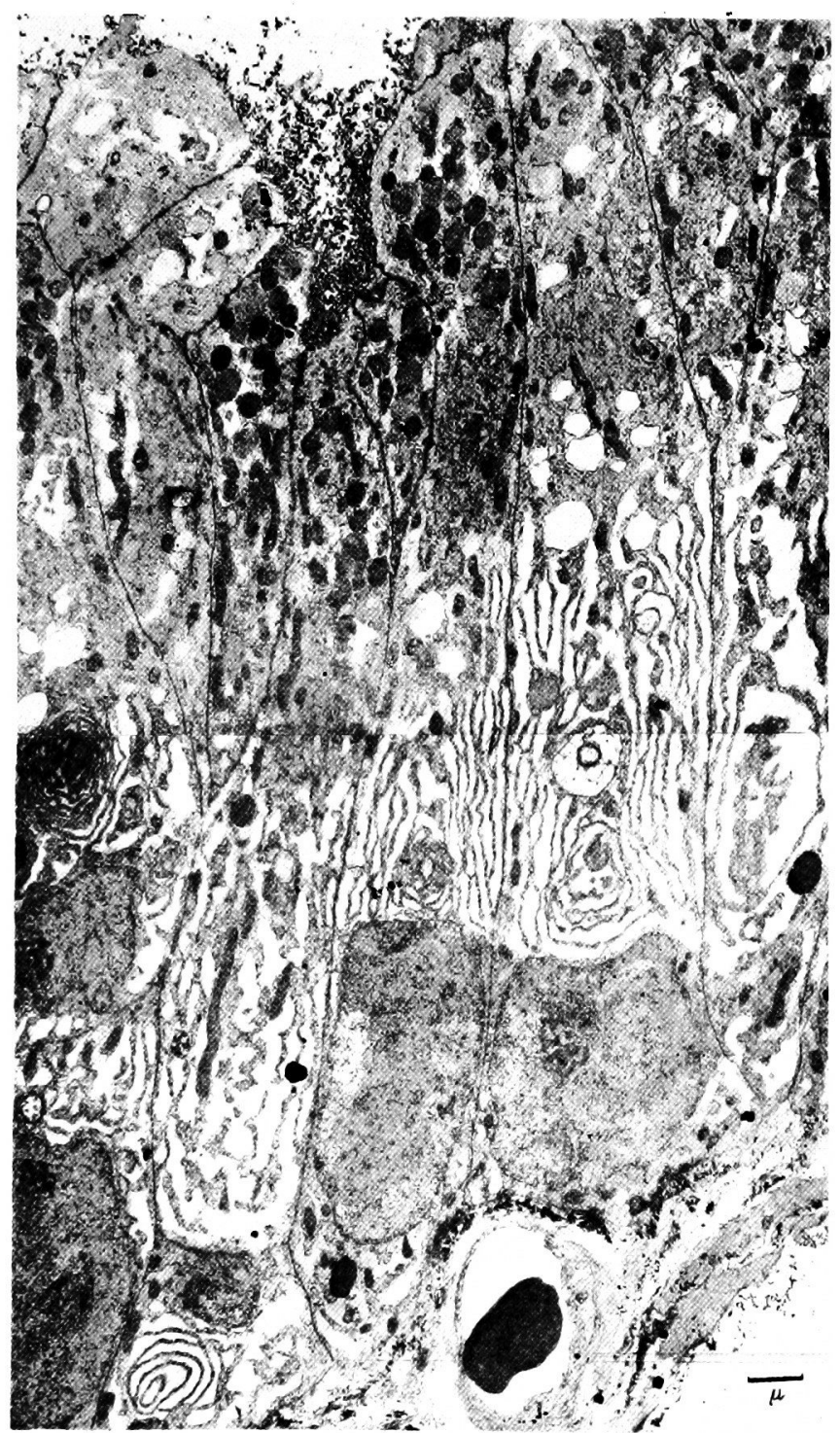

Fig. 9. Prostate administered $100 \mu \mathrm{g}$. of testosterone in 3 days soon after castration.

The cells are very tall. Well-developed endoplasmic reticulum in supranuclear portion and well-developed Golgi's complex above them. Round granules of varying electron density near the luminar border. 


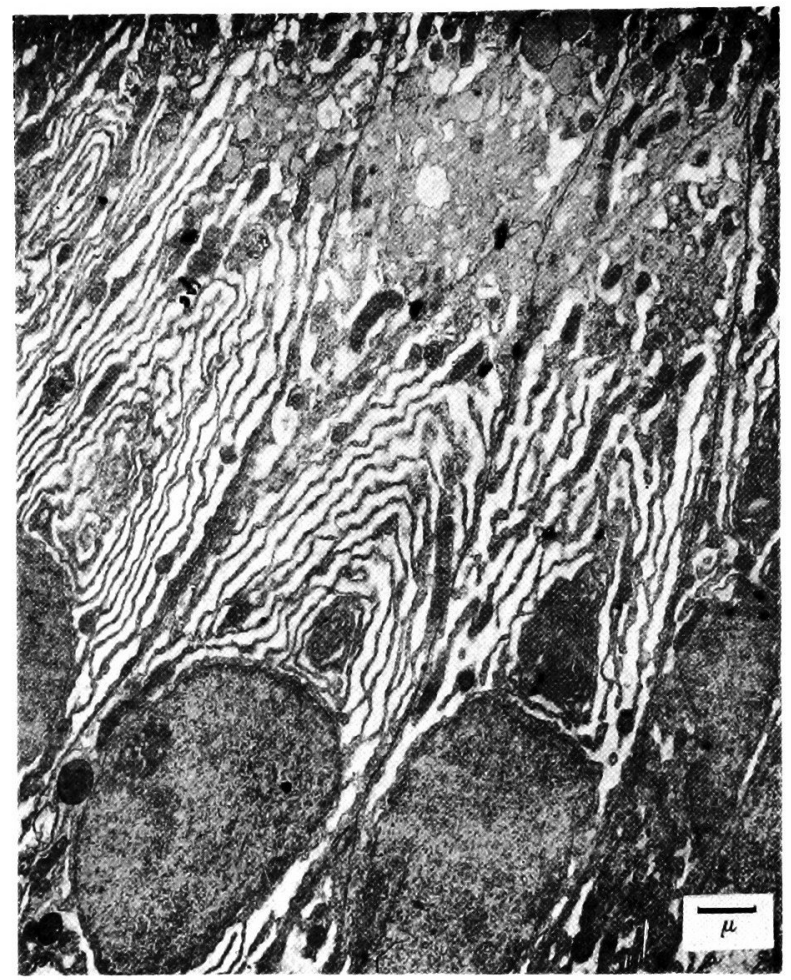

Fig. 10. Ditto.

Well-developed Golgi's complex and endoplasmic reticulum. 